\title{
A 1.8V 2.5-5.2 GHz CMOS Dual-input Two-stage Ring VCO
}

Wei-Hsuan Tu

Jyh-Yih Yeh

jhgf(a)aic.ee.ntu.edu.tw vjy@aic.ee.ntu.edu.tw
Hung-Chieh Tsai

Chorng-Kuang Wang

ckwang@cc.ee.ntu.edu.tw

Graduate Institute of Electronics Engineering and Department of Electrical Engineering, National Taiwan University, Taipei, Taiwan

\begin{abstract}
A $1.8 \mathrm{~V} 2.5$-to-5.2 GHz dual-input two-stage CMOS voltage-controlled ring oscillator (VCO) is presented. A novel delay cell used for the two-stage ring $\mathrm{VCO}$ is proposed to achieve low power dissipation and better phase noise performance. The delay cell adopts both inductive-peaking and shuntshunt feedback circuit techniques to obtain high gain and wide. Implemented in a $0.18 \mu \mathrm{m}$ CMOS technology using $1.8 \mathrm{~V}$ supply voltage, the $\mathrm{VCO}$ has a wide operating frequency range from $2.5 \mathrm{GHz}$ to $5.2 \mathrm{GHz}$ with coarse-tune VCO gain of $4.75 \mathrm{GHz} / \mathrm{V}$ and fine-tune VCO gain of $80 \mathrm{MHz} / \mathrm{V}$. At $3.6 \mathrm{GHz}$, the phase noise of the $\mathrm{VCO}$ is $-90.1 \mathrm{dBc} / \mathrm{Hz}$ at $1 \mathrm{MHz}$ frequency offset with low power consumption of $17 \mathrm{~mW}$. Because of the two-stage ring topology, the VCO can provide quadrature outputs. The total chip area is $0.4 \mathrm{~mm} \times 0.6 \mathrm{~mm}$.
\end{abstract}

\section{INTRODUCTION}

Voltage-controlled oscillators (VCOs) are critical building blocks in phase-locked loops (PLLs) and clock and data recovery circuits (CDRs), which are widely used in wireless and wire-line communication systems. Designing VCOs for monolithic integration is always desirable but most challenging. It has to achieve highfrequency operation with reasonable power consumption. In addition, the phase noise performance is also important. Finally, small chip area is essential to monolithic system integration.

In recent years, the CMOS LC-tank oscillators have shown an excellent phase noise performance with low power consumption because of a relatively high quality factor. However, the limited tuning range and large chip area have become critical drawbacks in LC VCOs. On the other hand, the ring VCOs exhibit several attractive features such as the wide frequency tuning range, the ease of integration with digital CMOS process, and the small chip area. Moreover, ring oscillators generate both in-phase and quadrature-phase outputs with an even number of delay cells.

The oscillation frequency of the ring oscillator is inversely proportional to the number of delay stages $\mathrm{N}$. Employing fewer delay stages can also reduce the power dissipation, chip area and cost. The minimum number of stages for quadrature outputs is $N=2$. Hence it is desirable to design a two-stage ring VCO. However, in order to satisfy Barkhausen's criteria, an additional phase shift is required in each delay cell.
Several novel delay cells have been proposed to compose the two-stage ring VCO [1][2], but much extra power is needed to provide an excess phase shift. The circuit described in [3] can achieve low power design, however the output signal swing is reduced in order to place the complex poles in front of the zero. Consequently, the phase noise performance of this twostage ring VCO is relatively poor. In the following, a $1.8 \mathrm{~V} 2.5-$ to-5.2 $\mathrm{GHz}$ dual-input two-stage ring $\mathrm{VCO}$ is presented. It will show that the two-stage ring VCO possesses many attractive features such as low power dissipation, large output signal swing, and wide tuning range.

The structure of this paper is as follows. In Section 2, the proposed delay cell is discussed. In Section 3 we then describe the complete two-stage ring VCO. The experimental results are shown in Section 4. Finally, a conclusion is given in Section 5.

\section{SHUNT-SHUNT FEEDBACK DELAY CELL}

The circuit schematic of the proposed delay cell is depicted in Fig. 1. The excess phase shift is provided by the shunt-shunt feedback configuration including M3 to

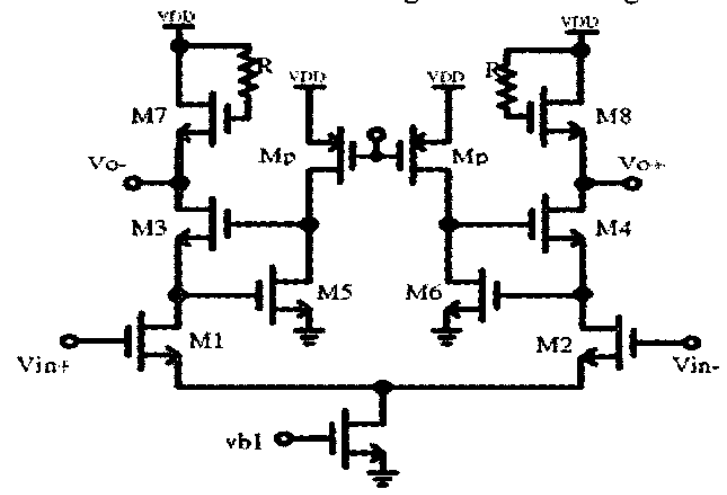

Fig. 1. Proposed delay cell

M6. Therefore, the loading device, containing a resistor and a NMOS, can be designed to exhibit the property of inductive peaking so that the ac small signal gain at the oscillation frequency and the output signal swing can be boosted. Although the total power dissipation seems to be increased due to the addition of two more current paths which are composed of M5, M6 and Mp's, the property of shunt-shunt feedback makes the required dc current associated with these two paths very small compared to that of the main signal paths. 
It is necessary to know the location of poles contributed by the shunt-shunt feedback configuration because it determines the amount of excess phase shift and hence the oscillation frequency. The circuit diagram and the corresponding small signal model shown in Fig.2 can be used to derive the location of poles in the

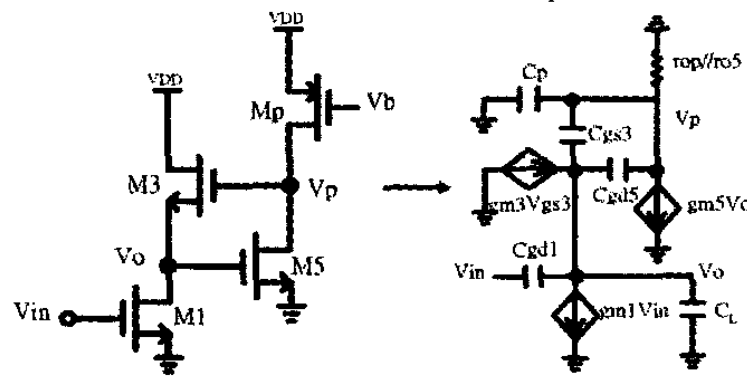

Fig. 2. Circuit diagram used to determine the poles in the feedback loop

feedback loop. By listing node equations, the transfer function from Vin to Vo can be derived as

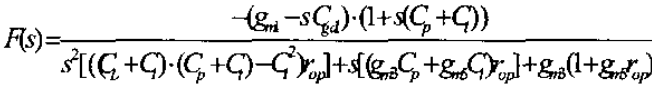

$$
\begin{aligned}
& \text { where } g_{m} r_{o p} \gg 1 \text { is assumed. }
\end{aligned}
$$

It can be observed from equation (1) that the location of the poles associated with the feedback loop can be very far without consuming much power due to the nature of the shunt-shunt feedback.

The overall transfer function $\mathrm{T}(\mathrm{s})$ from the input to the output of the delay cell can be expressed as the product of two transfer functions $T_{1}(s)$ and $T_{2}(s)$, where $T_{1}(s)$ represents the part of overall transfer function providing an excess phase shift and $\mathrm{T}_{2}(\mathrm{~s})$ explains the inductive-peaking behavior resulting from the loading devices.

$$
\begin{aligned}
& T(s)=\frac{v_{a}}{v_{i n}}=-T_{1}(s) \cdot T_{2}(s) \\
& g_{m} r_{o p} \cdot\left(\left(g_{o s}+\frac{1}{r_{o p}}\right)+s C_{p}\right)
\end{aligned}
$$

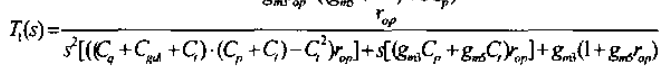

$$
\begin{aligned}
& T_{2}(s)=\frac{\left(1+C_{g s 8} R s\right)}{s^{2} C_{g s} C_{L} R+s\left(C_{L}+C_{g s 8}\right)+g_{m 8}}
\end{aligned}
$$

\section{TWO-STAGE RING VCO}

The tuning method adopted in this two-stage ring VCO is the delay interpolation tuning as shown in Fig. 3. This tuning method can achieve wide tuning range and relatively linear voltage-to-frequency characteristic. In

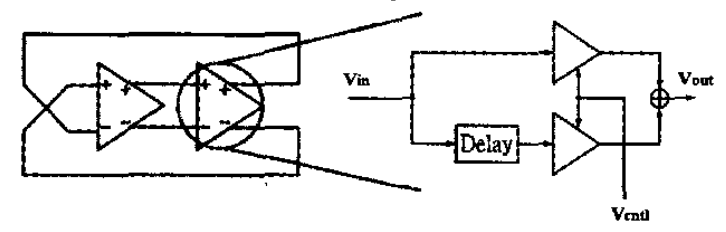

Fig. 3. Interpolation Tuning

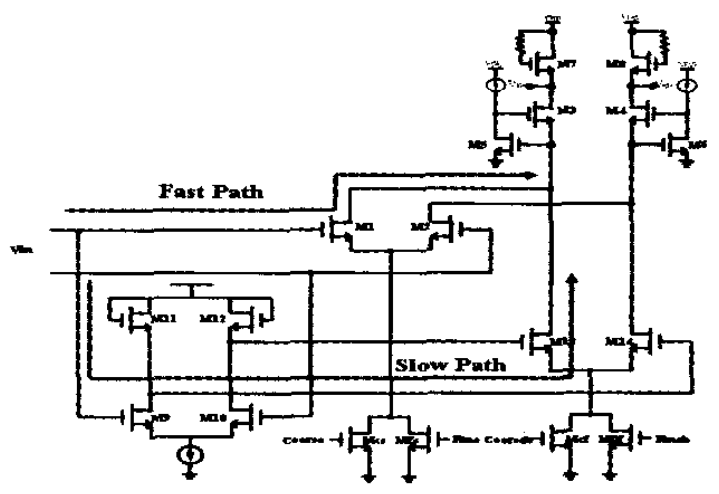

Fig. 4. Delay interpolation delay cell

addition, it is suitable for the fully differential operation in which the control lines of the VCO are also differential.

The circuit schematic of the complete delay stage is shown in Fig.4. The delay block shown in Fig.3 is implemented by M9 through M12. The fast and slow paths share the shunt-shunt feedback loop consisting of M3 to M6 and the inductive peaking loading devices containing M7, M8 and resistors. It should be noted that with the purpose of achieving both low jitter and wide capture range performance, modern PLLs and CDRs frequently use dual-loop architectures[4][5]. Hence the dual-input VCO is often desired. In order to decompose the VCO control line into coarse and fine tune inputs, the MOSs which are used to provide the tail current in each path are decomposed to two parallel MOSs. One is controlled by the fine tune input while the other is controlled by the coarse tune input. Finally, the current folding circuit [6] shown in Fig. 5 is used to alleviate the issue of limited voltage headroom. The current variation is performed through mirror arrangements driven by PMOS differential pairs M1-M2 and M3-M4.

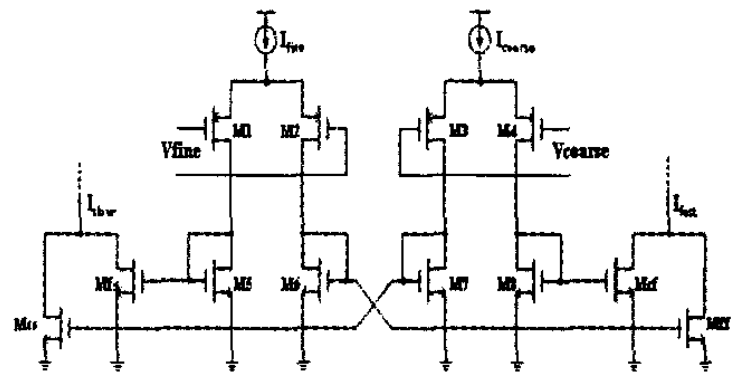

Fig. 5. Current folding circuit

\section{EXPERIMENTAL RESULTS}

The two-stage ring VCO is fabricated using $0.18 \mu \mathrm{m}$ CMOS technology and the chip photo is shown in Fig.6. The VCO covers $0.6 \times 0.4 \mathrm{~mm}^{2}$ chip area. The external components in the testing board include a variable resistor, and some decoupling capacitors. The measured transfer characteristics of the VCO are shown in Fig.7 


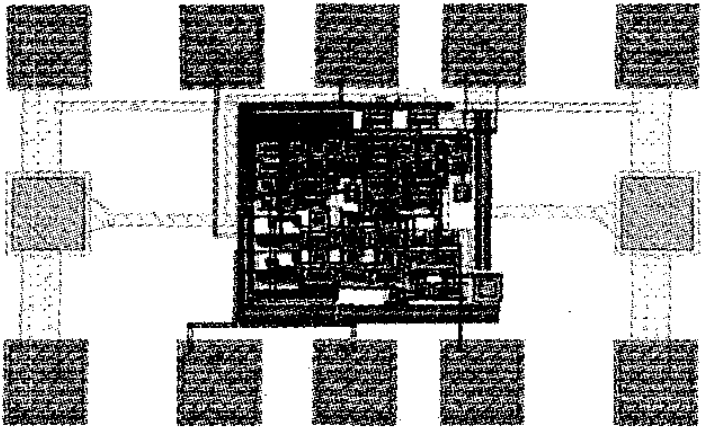

Fig. 6. Die photo of VCO

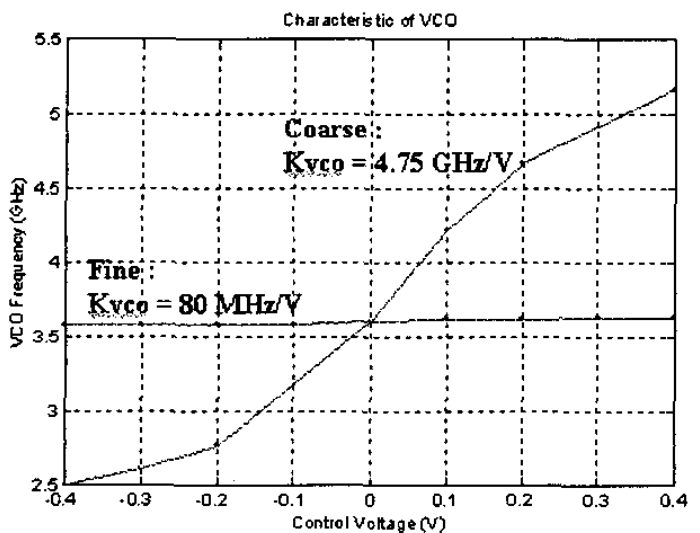

Fig. 7. Transfer characteristics of VCO

for both coarse and fine tuning. It can be seen that the gain of the coarse-tune control input is $4.75 \mathrm{GHz} / \mathrm{V}$, which is large enough to encompass process and temperature variations. On the other hand, the gain of the VCO with respect to the fine-tune control input is only $80 \mathrm{MHz} / \mathrm{V}$, which is quite small in order to reduce the VCO output jitter. The VCO has a wide operating frequency range from $2.5 \mathrm{GHz}$ to $5.2 \mathrm{GHz}$ which can be used in many applications. The overall tuning range of this two-stage ring VCO is about $74 \%$..

Finally, the phase noise performance of the freerunning two-stage VCO is measured as shown in fig. 8 .

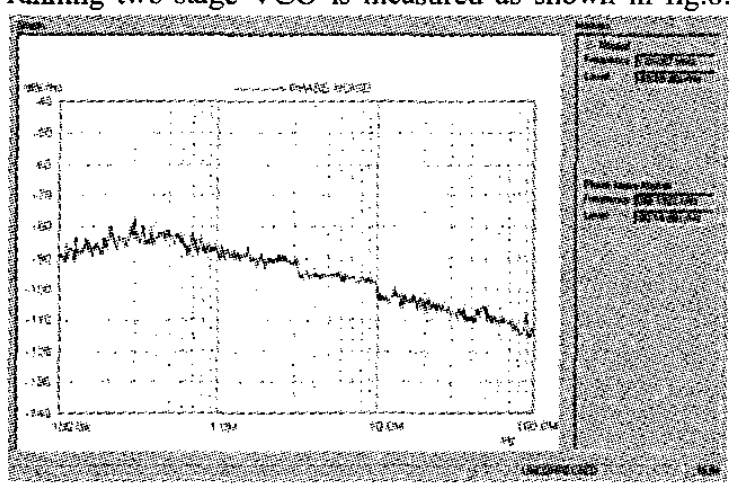

Fig. 8. Phase noise of VCO

Measured at $3.6 \mathrm{GHz}$, it shows that the phase noise of the $\mathrm{VCO}$ is $-90.1 \mathrm{dBc} / \mathrm{Hz}$ at $1 \mathrm{MHz}$ frequency offset. The total power consumption of the two-stage ring $\mathrm{VCO}$ is only $17 \mathrm{~mW}$ under $1.8 \mathrm{~V}$ supply voltage.

The performance summary of the dual-input twostage ring VCO is listed in Table 1.

\begin{tabular}{|c|c|}
\hline Oscillator Type & Two stage ring VCO \\
\hline Center Frequency & $3.6 \mathrm{GHz}$ \\
\hline Tuning Range & $74 \%$ \\
\hline Phase Noise & $4.75 \mathrm{GHz} / \mathrm{V}, 80 \mathrm{MHz} / \mathrm{V}$ \\
\hline I \& Q Output & $-90.1 \mathrm{dBc} / \mathrm{Hz} @ 1 \mathrm{MHz}$ offset \\
\hline Power Dissipation & Yes \\
\hline Chip Area & $17 \mathrm{~mW}$ \\
\hline Technology & CMOS $0.18 \mu \mathrm{m}$ with $1.8 \mathrm{~V}$ supply voltage \\
\hline
\end{tabular}

Table 1. Performance summary

In order to evaluate the performance of different ring oscillator topologies, a figure of merit (FoM) provided by [7] is used to compare different designs. The performance comparison results are given in Table 2. It can be seen that the two-stage ring VCO presented here has better FoM compared to [8]-[10] and has the same FoM with the design in [6]. However, the supply voltage of our design is only $1.8 \mathrm{~V}$ while the supply voltage in [6] has to be boosted to $2.5 \mathrm{~V}$ to guarantee correct operation.

\section{CONCLUSION}

In this paper, a $1.8 \mathrm{~V} 2.5$-to-5.2GHz dual-input twostage CMOS ring VCO is presented. A delay cell used in VCO adopts both inductive-peaking and shunt-shunt feedback circuit techniques to attain high gain, low power and wide bandwidth performance. By using interpolation tuning method, the VCO has a wide operating range of $74 \%$ with coarse-tune VCO gain of $4.75 \mathrm{GHz} / \mathrm{V}$ and fine-tune $\mathrm{VCO}$ gain of $80 \mathrm{MHz} / \mathrm{V}$. At $3.6 \mathrm{GHz}$, the phase noise of the $\mathrm{VCO}$ is $-90.1 \mathrm{dBc} / \mathrm{Hz}$ at $1 \mathrm{MHz}$ frequency offset. The two-stage ring $\mathrm{VCO}$ as presented in this paper occupies a $0.6 \mathrm{~mm} \times 0.4 \mathrm{~mm}$ chip area in a $0.18 \mu \mathrm{m} 1 \mathrm{P} 6 \mathrm{M}$ CMOS technology. The total power consumption of this chip is only $17 \mathrm{~mW}$ under a $1.8 \mathrm{~V}$ supply voltage. 
2004 IEEE Asia-Pacific Conference on Advanced System Integrated Circuits(AP-ASIC2004)/ Aug. 4-5, 2004

\begin{tabular}{|c|c|c|c|c|c|}
\hline & Proposed VCO & {$[6]$} & {$[8]$} & {$[9]$} & {$[10]$} \\
\hline $\begin{array}{c}\text { Center } \\
\text { Frequency }\end{array}$ & $3.6 \mathrm{GHz}$ & $5 \mathrm{GHz}$ & $5 \mathrm{GHz}$ & $5 \mathrm{GHz}$ & $1.56 \mathrm{GHz}$ \\
\hline $\begin{array}{c}\text { Tuning } \\
\text { Range }\end{array}$ & $74 \%$ & $54 \%$ & $24 \%$ & $130 \%$ & $12 \%$ \\
\hline $\begin{array}{c}\text { Phase Noise @ } \\
\text { 1MHz offset }\end{array}$ & $-90.1 \mathrm{dBc} / \mathrm{Hz}$ & $-88.2 \mathrm{dBc} / \mathrm{Hz}$ & $-88.3 \mathrm{dBc} / \mathrm{Hz}$ & $-82 \mathrm{dBc} / \mathrm{Hz}$ & $-97.4 \mathrm{dBc} / \mathrm{Hz}$ \\
\hline \begin{tabular}{c} 
Supply Voltage \\
\hline $\begin{array}{c}\text { Power } \\
\text { Dissipation }\end{array}$
\end{tabular} & 1.8 & 2.5 & 1.8 & 1.8 & 1.8 \\
\hline Technology & $\mathrm{CMOS} 0.18 \mathrm{~mm}$ & $\mathrm{CMOS} 0.18 \mathrm{um}$ & $\mathrm{CMOS} 0.18 \mathrm{um}$ & $\mathrm{CMOS} 0.18 \mathrm{um}$ & $\mathrm{CMOS} 0.18 \mathrm{um}$ \\
\hline FoM & 178.9 & 178.9 & 177.4 & 164.7 & $48.2 \mathrm{~mW}$ \\
\hline
\end{tabular}

Table 2. Performance comparison

\section{REFERENCES}

[1] Hormoz Djahanshahi and C.Andre T, "Differential CMOS Circuit for $622 \mathrm{MHz} / 933 \mathrm{MHz}$ CDR Application," IEEE J. Solid-State Circuits, June 2000.

[2] William Shing Tak Yan, "A $900 \mathrm{MHz}$ CMOS Low Phase Noise Voitage Controlled Ring Oscillator," IEEE Trans. on Circuits and Systems, Feb. 2001.

[3] Seema Butala Anand and Behzad Razavi, "A CMOS Clock Recovery Circuit for 2.5-Gb/s NRZ Data," IEEE J. SolidState Circuits, Mar. 2001.

[4] J. Christoph Scheytt, "A 0.155, 0.622, and $2.488 \mathrm{~Gb} / \mathrm{s}$ Automatic Bit Rate Selecting CDR IC for Bit Rate Transparent SDH System," ISSCC Dig. Tech, Feb. 1999.

[5] H. Noguchi, T. Tateyama, and M. Dkamoto et al., "A 9.9G$10.8 \mathrm{~Gb} / \mathrm{s}$ Rate-Adaptive CDR with No Extemal Reference Clock for WDM Optical Fiber Transmission," ISSCC Dig. Tech. Papers, Feb. 2002.

[6] Jafar Savoj and Behzad Razavi, "A 10Gb/s CMOS CDR Circuit with a Half-Rate Linear Phase Detector", IEEE J. Solid-State Circuits, May 2001.

[7] P. Kinget, "Integrated GHz voltage controlled oscillators", Proc. AACD Workshop, Nice, March 1999.

[8] Jafar Savoj and Behzad Razavi, "A 10Gb/s CMOS Clock and Data Recovery Circuit with Frequency Detection," ISSCC Dig. Tech. Papers, Feb. 2001.

[9] Afshin Rezayee and Ken Martin, "A Coupled Two-Stage Ring Oscillator." IEEE 2001.

[10] S.P. Chen, "Designt and Implementation of a $3.125 \mathrm{~Gb} / \mathrm{s}$ Clock and Data Recovery Circuit," M.S. Thesis, National Taiwan Univ., 2003. 